Pacific Journal of Mathematics

RADIAL AVERAGING TRANSFORMATIONS WITH VARIOUS 


\section{RADIAL AVERAGING TRANSFORMATIONS WITH VARIOUS METRICS}

\section{Catherine Bandle and Moshe Marcus}

In [5] one of the authors introduced the notion of a radial averaging transformation of domains in the plane, which was based on the metric given by the line element $d s^{2}=$ $\left(1 / r^{2}\right)\left(d x_{1}^{2}+d x_{2}^{2}\right)$ where $\left(x_{1}, x_{2}\right)$ are the cartesian and $(r, \theta)$ are the polar coordinates. This transformation is useful in obtaining estimates for conformal capacity of condensers and for conformal radius of domains. In this paper we discuss averaging transformations in $m$-dimensional spaces $(m \geqq 2)$, based on various metrics of the form $d s^{2}=g^{2}(r) \sum_{i=1}^{m}\left(d x_{i}\right)^{2}$, where $g(r)$ is a positive, continuous function of $r(0<r<\infty)$.

With the help of these transformations we are able to obtain estimates for energy integrals of the form

$$
\int_{\Omega}|\nabla F|^{2} g(r) r^{3-m} d x \quad\left(d x=d x_{1} d x_{2} \cdots d x_{m}\right) .
$$

These estimates can be used to compare capacities of different condensers filled with nonhomogeneous dielectric [cf. Kühnau [3] and the literature cited there]. As a further application we derive inequalities for conformal capacity and conformal radius in the plane and similar results in higher dimensional spaces. In this direction we have results for the case where $g(r)=r^{\beta} \beta \geqq m-3$. They include the symmetrization results obtained by Szegö in [7]. The method presented seems to be quite general, and we believe that it might be employed also with other classes of metrics $g$.

1. Estimates for energy integrals. Let $g(r)$ be a positive continuous function for $0<r<\infty$ and let $G(r)$ be a primitive of $g$. $\left(r, \theta_{1}, \cdots, \theta_{m-1}\right)$ are the polar coordinates defined in the following way:

$$
\begin{gathered}
x_{1}=r \cos \theta_{1}, x_{2}=r \sin \theta_{1} \cos \theta_{2}, x_{3}=r \sin \theta_{1} \sin \theta_{2} \cos \theta_{3}, \\
\ldots \ldots \cdot \\
x_{n}=r \sin \theta_{1} \sin \theta_{2} \cdots \sin \theta_{m-2} \sin \theta_{m-1}
\end{gathered}
$$

where $0 \leqq \theta_{i} \leqq \pi$ for $i=1, \cdots, m-2$ and $-\pi \leqq \theta_{m-1} \leqq \pi$. Let $\rho$ be a fixed positive number and set:

$$
\left\{\begin{array}{l}
u=G(r)-G(\rho) \\
v_{i}=\theta_{i}
\end{array} \quad i=1, \cdots, m-1 .\right.
$$

Let $\Omega$ be an open set in $R^{m}$ which does not contain the sphere $\{x ;|x| \leqq \rho\}$ and the hyperplanes $\theta_{i}=0 i=1,2, \cdots, n-2$. Then for $F(x) \in C^{1}(\Omega)\left[x=\left(x_{1}, x_{2}, \cdots, x_{m}\right)\right]$ we have 


$$
|\nabla F|^{2}=F_{r}^{2}+\sum_{i=1}^{m-1} r^{-2} f_{i} F_{\theta_{i}}^{2}
$$

where $f_{i}=f_{i}\left(\theta_{1}, \theta_{2}, \cdots, \theta_{i-1}\right)$ if $i=2,3, \cdots, m-1$ and $f_{1}=1$.

If $d \omega=\Phi\left(\theta_{1}, \cdots, \theta_{m-2}\right) d \theta\left[d \theta=d \theta_{1}, \cdots, d \theta_{m-1}\right]$ denotes the surface element of the unit sphere, then the volume element $d x$ is given by $d x=\left(r^{m-1} / g(r)\right) d u d \omega$. Hence

$$
\int_{\Omega}|\nabla F|^{2} g(r) r^{3-m} d x=\int_{\tilde{\Omega}}\left[\widetilde{F}_{u}^{2} r^{2} g^{2}(r)+\sum_{i=1}^{m-1} f_{i} \widetilde{F}_{v_{i}}^{2}\right] d u d \omega
$$

where $\widetilde{\Omega}$ is the image of $\Omega$ by (1.1), and

$$
\widetilde{F}\left(u, v_{1}, \cdots, v_{m-1}\right)=F\left(x_{1}(r, \theta), \cdots, x_{n}(r, \theta)\right)
$$

where $\theta=\left(\theta_{1}, \cdots, \theta_{m-1}\right)$.

We denote

$$
q^{2}=r g(r)
$$

DEFINITION 1.1. Let $D$ be an open set containing $\{x ;|x| \leqq \rho\}$ and $D_{\rho}=D-\{x ;|x| \leqq \rho\}$. Denote

$$
l(\theta)=\int_{E_{\theta}} g(r) d r,
$$

where

$$
E_{\theta}=D_{\rho} \cap\{x=(r, \tilde{\theta}) ; \tilde{\theta}=\theta\}
$$

we define

$$
R(\theta)=G^{-1}[l(\theta)+G(\rho)] .
$$

Clearly $R(\theta)$ does not depend on $\rho$. Let

$$
\begin{aligned}
D^{*}= & \left\{x=(r, \theta) ; 0 \leqq r<R(\theta), 0 \leqq \theta_{i} \leqq \pi i=1, \cdots, m-2,\right. \\
& \left.-\pi \leqq \theta_{m-1} \leqq \pi\right\}
\end{aligned}
$$

The transformation $D \rightarrow D^{*}$ will be called the radial concentration with metric $g$.

ExAmple. Suppose that $D$ is compact and that the rays $\theta=$ constant intersect $\partial D$ in a finite number of points $r_{1}(\theta)<r_{2}(\theta)<\cdots<$ $r_{2 \nu+1}(\theta)$. In the case where $g(r)=r^{\beta-1}$, we have

$$
R(\theta)= \begin{cases}\left\{r_{1}^{\beta}+\sum_{j=1}^{2 \nu}\left(r_{2 j+1}^{\beta}-r_{2 j}^{\beta}\right)\right\}^{1 / \beta} & \text { if } \beta \neq 0 \\ r_{1} \prod_{j=1}^{2 \nu} \frac{r_{2 j+1}}{r_{2 i}} & \text { if } \beta=0 .\end{cases}
$$


REMARK. If $\widetilde{D}_{\rho}$ is the image of $D_{\rho}$ by $(1.1)$ and $\widetilde{D}_{\rho}^{*}$ is the image of $D_{\rho}^{*}$ by (1.1), then $\widetilde{D}_{\rho}^{*}$ is obtained from $\widetilde{D}_{\rho}$ by:

$$
\begin{aligned}
& \widetilde{D}_{\rho}^{*}=\left\{(u, v) \mid 0<u<l(v), 0 \leqq v_{i} \leqq \pi \quad \text { if } \quad i=1, \cdots, n-2\right\} \\
& -\pi \leqq v_{n-1} \leqq \pi
\end{aligned}
$$

where $v=\left(v_{1}, \cdots, v_{n-1}\right)$ and

$$
l\left(v_{0}\right)=\text { linear measure of }\left(\widetilde{D}_{\rho}^{*} \cap\left\{v=v_{0}\right\}\right) .
$$

DeFinition 1.2. Let $D$ be a domain which contains $|x| \leqq \rho$. Let $F(x)$ be a continuous function in $R^{m}$ such that $F \equiv 0$ outside $D$ and $F \equiv 1$ in a compact subset of $D$ (denoted by $E$ ) such that $\{|x| \leqq \rho\} \subset E$. Suppose that in $\Omega=D-E, 0<F<1$ and that on every ray through the origin $F$ takes every value $\lambda,(0<\lambda<1)$, only a finite number of times. Let

$$
D_{\lambda}(F)=\{(x) \mid F(x)>\lambda\}, \quad(0 \leqq \lambda<1) .
$$

Let $D_{\lambda}^{*}$ be the $g$-radial concentration of $D_{\lambda}$ and let $F^{*}$ be defined as follows:

$$
F^{*}= \begin{cases}1 & \text { in } E^{*} \\ \lambda & \text { on the boundary of } D_{\lambda}^{*}, \quad 0<\lambda<1 \\ 0 & \text { outside } D^{*} .\end{cases}
$$

(Here $E^{*}$ is defined as in Definition 1 except that in (1.7) $0 \leqq r \leqq R(\theta)$.) Then $F^{*}$ will be called the radial concentration of $F$, with metric $g$.

The following results are proved exactly in the same way as in [5]:

(i) $D^{*}$ is a starlike domain.

(ii) $E^{*}$ is a compact, connected, starlike set.

(iii) If $F$ is continuous then $F^{*}$ is continuous.

(iv) If $F$ is continuous in $R^{m}$ and Lip in every compact subset of $D-E$, then $F^{*}$ has the same properties with respect to $D^{*}-E^{*}$.

Also the following basic result is obtained by essentially the same method as in [5].

LEMma 1.1. Let $D, \Omega, F$ be as in Definition 1.2. Suppose also that $F \in C^{1}(\Omega)$, that $F \in C^{0}$ in $R^{m}$, and that on every ray $\theta=$ constant, the set of points in $\Omega$ where $(\partial F / \partial r)=0$ is at most a finite set. Finally suppose that:

$$
p(u)=[r g(r)]_{r=r(u)} \text { is convex or monotone. }
$$

Then we have:

$$
\int_{\Omega^{*}}\left|\nabla F^{*}\right|^{2} g(r) r^{3-m} d x \leqq \int_{\Omega}|\nabla F|^{2} g(r) r^{3-m} d x
$$


where $\Omega^{*}=D^{*}-E^{*}, \Omega=D-E$.

We shall sketch the main ideas of the proof for the case $\Omega \subset R^{2}$. It is not difficult to see that the same proof works also for $\Omega \subset R^{m}(m>2)$.

Proof. Consider the level line $\widetilde{F}(u, v)=\lambda$ where $0<\lambda<1$ and $v=v_{1}$. Except for a finite number of $\lambda$ the rays $v=$ constant intersect the level line in a finite number of points $\left(u_{j}, v\right)$ with

$$
u_{1}(\lambda, v)<u_{2}(\lambda, v)<\cdots<u_{2 \nu+1}(\lambda, v) .
$$

Actually we shall show that not only the total energy integral is diminished by radial concentration but even each of its infinitesimal parts between two level lines given by

$$
\widetilde{F}(u, v)=\lambda \text { and } \widetilde{F}(u, v)=\lambda+d \lambda .
$$

Introducing $\lambda, v$ as new variables we find that this infinitesimal part is given by

$$
\int_{v=-\pi}^{\pi}\left(\sum_{j=1}^{2 \nu+1}\left\{\frac{p^{2}\left(u_{j}\right)}{\left|\partial u_{j} / \partial \lambda\right|}+\frac{\left(\partial u_{j} / \partial v\right)^{2}}{\left|\partial u_{j} / \partial \lambda\right|}\right\}\right) d \lambda d v .
$$

Because of the Schwarz inequality, (1.14) is greater than

$$
\int_{v=-\pi}^{\pi} \frac{\left[\sum_{j=1}^{2 \nu+1} p\left(u_{j}\right)\right]^{2}+\left[\sum_{j=1}^{2 \nu+1}\left|\partial u_{j} / \partial v\right|\right]^{2}}{\sum_{j=1}^{2 \nu+1}\left|\partial u_{j} / \partial \lambda\right|} d \lambda d v .
$$

From the monotonicity or convexity of $p(u)$ it follows [cf. 8]

$$
p\left(\sum_{j=1}^{2 \nu+1}(-1)^{j+1} u_{j}\right) \leqq \sum_{j=1}^{2 \nu+1} p\left(u_{j}\right) .
$$

Thus, by Minkowski's inequality we have that (1.14) is greater than

$$
\int_{v=-\pi}^{\pi} \frac{\left[p\left(\sum_{j=1}^{2 \nu+1}(-1)^{j+1} u_{j}\right)\right]^{2}+\left.\left|\sum_{j=1}^{2 \nu+1}(-1)^{j+1} \partial u_{j} / \partial v\right|\right|^{2}}{\sum_{j=1}^{2 \nu+1}(-1)^{j+1} \partial u_{j} / \partial \lambda} d \lambda d v .
$$

Since $\left(\partial u_{j} / \partial \lambda\right)$ has alternating signs, the denominator of (1.15) does not vanish. The assertion follows immediately from (1.15). We now define the radial averaging transformation with metric $g$ in the same way as it was defined in [5] for the logarithmic measure.

DeFinition 1.3. Let $\left\{D_{1}, \cdots, D_{n}\right\}=\mathscr{D}$ be a family of open sets in $R^{m}$, each containing the sphere $|x| \leqq \rho(\rho$ arbitrary real number). 
Let $A=\left\{a_{j}\right\}_{j=1}^{n}$ where $a_{j}>0$ and $\sum_{j=1}^{n} a_{j}=1$. Let $l_{j}(\theta)$ be defined as in Definition 1.1 for $D_{j}$. Then set

$$
\begin{gathered}
l^{*}(\theta)=\sum_{j=1}^{n} a_{j} l_{j}(\theta), \\
R^{*}(\theta)=G^{-1}\left[l^{*}(\theta)+G(\rho)\right],
\end{gathered}
$$

and finally define $D^{*}$ as in (1.7) with $R(\theta)$ replaced by $R^{*}(\theta)$. We shall denote $D^{*}=\mathscr{R}_{g, A}(\mathscr{D})$, and call the transformation $\mathscr{D} \rightarrow D^{*}$ the radial averaging transformation with metric $g$.

In the special case where $D_{i} i=1,2, \cdots, n$ are obtained from a fixed domain $D$ by a combination of simple transformations such as rotations around the origin and reflections with respect to a plane through the origin, then the transformation $R_{A, g}$ becomes a symmetrization. For example, in $R_{3}$ with $g=1$ and $a_{j}=1 / n,(j=1, \cdots, n)$, we obtain, by rotations, the symmetrization given by:

$$
R^{*}\left(\theta_{1}, \theta_{2}\right)=\frac{1}{n} \sum_{j=1}^{n} R\left(\theta_{1}+\beta_{j}, \theta_{2}+\gamma_{j}\right),
$$

where $\beta_{j}, \gamma_{j}$ are arbitrary numbers. $D^{*}$ is defined as in (1.7) with $R$ replaced by $R^{*}$.

It might be observed that the radial concentration (1.7) is a particular case of the radial averaging transformation, i.e., the case $n \equiv 1$.

Definition 1.4. Let $\mathscr{D}$ and $A$ be defined as above. Suppose that $D_{j}$ is bounded. Let $E_{j}$ be a compact subset of $D_{j}$, which contains $\{|x| \leqq \rho\}$. Let $\mathscr{F}=\left\{F_{1}, \cdots, F_{n}\right\}$ be a set of functions such that each $F_{j}$ has the properties described in Definition 1.2 with respect to $D_{j}$ and $E_{j}$. We define $D_{\lambda}\left(F_{j}\right)$ as in (1.10) and

$$
D_{\lambda}^{*}=\mathscr{R}_{g, A}\left(D_{\lambda}\left(F_{1}\right), \cdots, D_{\lambda}\left(F_{n}\right)\right) \text {. }
$$

Finally we define $F^{*}$ as in (1.12). The transformation $\mathscr{F} \rightarrow F^{*}$ will be called a radial averaging transformation on $\mathscr{F}$ with metric $g$. We shall denote $F^{*}=\mathscr{R}_{g, A}(\mathscr{F})$.

The analogous properties to (i)-(iv) for the radial averaging transformation are verified exactly as in [5]. Also the following result is proved essentially in the same way as the parallel result in [5].

THeorem 1.1. Let $\mathscr{D}, \mathscr{F}$ be as in Definition 1.4. Suppose also that each $F_{j}$ has the properties described in Lemma 1.1 with respect to $D_{j}, E_{j}$. Finally suppose that $p(u)$ (defined as in Lemma 1.1) is convex. Then we have:

$$
\int_{\Omega^{*}}\left|\nabla F^{*}\right|^{2} \frac{g(r)}{r^{m-3}} d x \leqq \sum_{j=1}^{n} a_{j} \int_{\Omega_{j}}\left|\nabla F_{j}\right|^{2} \frac{g(r)}{r^{m-3}} d x
$$


where $D^{*}=\mathscr{R}_{g, A}(\mathscr{D}), E^{*}=\mathscr{R}_{g, A}\left(E_{1}, \cdots, E_{n}\right) \Omega^{*}=D^{*}-E^{*}, \Omega_{j}=D_{j}-E_{j}$,.

REMARKS (1) Lemma 1.1 is contained in Theorem 1.1 for the particular case $n=1$.

(2) Szegö [7] proved (1.18) for $g(r)=r^{m-3}$ and $a_{j}=1 / n$ $j=1, \cdots, n$ and for $D_{j}$ and $E_{j} j=1, \cdots, n$ obtained from $D$ and $E$ by certain rotations. He assumed further that $\Omega$ is starlike and that $F$ has starlike level surfaces.

(3) If $g(r)=1 / r$ (i.e., the logarithmic metric) and $m=2$, then the results obtained here coincide with the results of $[5,4]$. In this case we can obtain from (1.18) inequalities for the (conformal) capacities of cylindrical condensers with a homogeneous dielectric. By the same method we can derive from (1.18) inequalities for capacities of condensers with an inhomogeneous dielectric.

2. Estimates for capacities. In this section we describe a method by which the results of Theorem 1.1, with various metrics $g$, can be used to derive inequalities for condensers with homogeneous dielectrics.

Let $D$ be a bounded domain and $E$ a compact subset of $D$ which contains the sphere $\{|x| \leqq \rho\}$. We denote as usual $\Omega=D-E$. We assume that the boundary of $\Omega$ is sufficiently smooth so that Green's theorem may be used. Let $C$ be the "inner boundary" of $\Omega$, i.e., $\bar{\Omega} \cap E$.

Consider a function $\omega$ which is continuous in $R^{m}$ such that $\omega \in C^{1}(\Omega), \omega \equiv 0$ outside $D$ and $\omega \equiv 1$ in $E$. Let $h$ be defined in $\Omega$ by $h=\omega / q$, where $q$ is a positive function of $r(0<r<\infty)$ such that $q \in C^{2}(0, \infty)$. Because of the identity

$$
(\operatorname{grad} u v)^{2}=u^{2} \operatorname{grad}^{2} v+\operatorname{div}\left(v^{2} u \operatorname{grad} u\right)-v^{2} u \Delta u
$$

we have

$$
\int_{\Omega}|\nabla \omega|^{2} d x=\int_{\Omega}|\nabla h|^{2} q^{2} d x-\int_{\Omega} h^{2} q \Delta q d x-\oint \frac{\omega^{2}}{q} \frac{\partial q}{\partial n} d x
$$

[ $n$ inner normal, $d s$ surface element of $C$ ].

We now restrict our attention to the case where $E=\{|x| \leqq \rho\}$ in which case $C$ is the sphere $|x|=\rho$. We also assume that $\omega$ is harmonic in $\Omega$ and that $q(r)$ is analytic for $0<r<\infty$.

Let us apply the transformation of radial concentration with metric $g$, where $q^{2}=r g(r)$, to $D$ and $h$. We denote the resulting domain and function by $D^{*}, h^{*}$ respectively and we set $\Omega^{*}=D^{*}-E$. (In this case $E^{*}=E$.) It is easily verified that

$$
\int_{\Omega} h^{2} g(r) r^{1-m} d x=\int_{\Omega^{*}} h^{* 2} g(r) r^{1-m} d x .
$$


Now suppose that $q$ is chosen in such a manner that:

(2.3) (i ) $q \Delta q=c g(r) r^{1-m}$ where $q^{2}=r^{3-m} g(r)$ and $c$ is an arbitrary constant.

(ii) $q$ is positive and nondecreasing.

(iii) $p(u)=[r g(r)]_{r=r(u)}$ is convex (where $r(u)=G^{-1}[u+G(\rho)]$ see (1.1)).

Since $\omega$ is harmonic in $\Omega$ we have $0<\omega<1$ in $\Omega$ and since $q$ is nondecreasing $0<h<(1 / q(\rho))$ in $\Omega$ with $h=(1 / q(\rho))$ on $C$ and $h=0$ on the boundary of $D$. Furthermore since $h$ is an analytic function of $r$ on the intersection of any ray $\Omega=$ constant with $\Omega$, it is clear that $h$ satisfies all the assumptions of Lemma 1.1 (if $\Omega$ has a smooth boundary). Hence we obtain:

$$
\int_{\Omega^{*}}\left|\nabla h^{*}\right|^{2} q^{2} d x \leqq \int_{\Omega}|\nabla h|^{2} q^{2} d x
$$

By (2.1), (2.2), (2.3), and (2.4) we get

$$
\int_{\Omega}|\nabla \omega|^{2} d x \geqq \int_{\Omega^{*}}\left|\nabla h^{*}\right|{ }^{2} q^{2} d x-\int_{\Omega^{*}} h^{* 2} q \Delta q d x-\oint_{C} \frac{1}{q} \frac{\partial q}{\partial n} d s .
$$

But, again by (2.1), the right-hand side of (2.5) is equal to:

$$
\int_{\Omega^{*}}\left|\nabla \omega^{* *}\right|^{2} d x
$$

where $\omega^{* *}=h^{*} q$; note that $\omega^{* *}=1$ on $C$ and $\omega^{* *}=0$ on the boundary of $D^{*}$. Also, since $h^{*}$ is Lip in every compact subset of $\Omega^{*}$, so is $\omega^{* *}$. Hence $\omega^{* *}$ is an admissible function for the variational definition of the capacity of the condenser $\Omega^{*}$; if $\omega^{\prime}$ is harmonic in $\Omega^{*}$ and $\omega^{\prime}=1$ on $C$ and $\omega^{\prime}=0$ on the boundary of $D^{*}$, then:

$$
I\left(\Omega^{*}\right)=\int_{\Omega^{*}}\left|\nabla \omega^{\prime}\right|^{2} d x \leqq \int_{\Omega^{*}}\left|\nabla \omega^{* *}\right|^{2} d x,
$$

where $I\left(\Omega^{*}\right)$ is the capacity of $\Omega^{*}$. (As a reference for the facts quoted here see for instance [1] and [6].) From (2.5) and (2.6) we finally obtain

$$
I\left(\Omega^{*}\right) \leqq I(\Omega),
$$

where $I(\Omega)$ is the m-dimensional capacity of $\Omega$.

To sum up this result we state

LEMma 2.1. Let $D$ be a bounded domain in $R^{m}$ containing the sphere $\{|x|<\rho\}$. Let $\Omega=D-\{|x| \leqq \rho\}$. Let $q(r)$ be a positive analytic function of $r$ for $0<r<\infty$, satisfying (2.3). Let $D^{*}$ denote the domain obtained by radial concentration with metric $g$ from the domain $D$. We assume that $D^{*}$ is not the entire space $R^{m}$. Then 


$$
I\left(\Omega^{*}\right) \leqq I(\Omega)
$$

where $\Omega^{*}=D^{*}-\{|x| \leqq \rho\}$.

REMARK. In the previous discussion we assumed that the boundary of $D$ is smooth; but the result of Lemma 2.1 is obtained for general domains $D$ by the standard method of approximating a given domain by a sequence of domains with smooth boundary.

Using a result of Pólya-Szegö [6] on the connection between capacity and conformal radius, the following result is obtained as an immediate consequence of Lemma 2.1:

LEMma 2.2. Let $D$ be a domain in the plane containing the origin and let $D^{*}$ be the domain obtained from $D$ by radial concentration with metric $g$. Suppose that $g$ is analytic for $0<r<\infty$ and satisfies (2.3). Denote by $r_{0}$ (resp. $r_{0}^{*}$ ) the conformal radius of $D$ (resp. $\left.D^{*}\right)$ at the origin. (We assume that $D^{*}$ is not the entire plane.) Then:

$$
r_{0} \leqq r_{0}^{*} \text {. }
$$

By the same arguments used in the proof of Lemma 2.1, one obtains the following result (based on Theorem 1.1):

Theorem 2.1. Let $\mathscr{D}=\left\{D_{1}, \cdots, D_{n}\right\}$ be a family of domains in $R^{m}$ each of which does not contain $z=\infty$ and contains the sphere $|x| \leqq \rho$. Let $q$ be a positive analytic function of $r$ for $0<r<\infty$, satisfying (2.3). Let $D^{*}=\mathscr{R}_{g, A}(\mathscr{D})$ and suppose that $D^{*}$ is not the entire space. Denote: $\Omega_{j}=D_{j}-\{|x| \leqq \rho\} \Omega^{*}=D^{*}-\{|x| \leqq \rho\}$. Then:

$$
I\left(\Omega^{*}\right) \leqq \sum_{j=1}^{n} a_{j} I\left(\Omega_{j}\right)
$$

In the particular case where $g(r)=r^{m-3}$, (2.10) holds for general condensers $\Omega_{j}=D_{j}-E_{j},(j=1, \cdots, n)$, where $E_{j}$ is a compact subset of $D_{j}$ containing the sphere $|x| \leqq \rho$. (In this case, $\Omega^{*}=D^{*}-E^{*}$ where $E^{*}=\mathscr{R}_{g, A}\left(E_{1}, \cdots, E_{n}\right)$.)

The last statement of the theorem is an immediate consequence of Theorem 1.1, since in this case $q=g(r) / r^{m-3} \equiv 1$.

Again, applying the result of Pólya-Szegö [6] mentioned above (see also Hayman [1, p. 82]) we obtain from Theorem 2.1 (with $m=2$ ):

Theorem 2.2. Let $\mathscr{D}=\left\{D_{1}, \cdots, D_{n}\right\}$ be a family of domains in the plane containing the origin and let $D^{*}=\mathscr{R}_{,_{A}}(\mathscr{D})$ where $g(r)$ is positive and analytic for $0<r<\infty$ and satisfies (2.3). Denote by $r_{0, j}$ (resp. $r_{0}^{*}$ ) the conformal radius of $D_{j}$ (resp. $D^{*}$ ) at the origin. (We assume that $D^{*}$ is not the entire plane.) Then: 


$$
\min \left(r_{0,1}, \cdots, r_{0, n}\right) \leqq r_{0}^{*} \text {. }
$$

A family of functions $q$ which satisfy (2.3) (i) is established. It depends on the constant $c$.

(a) Let $c>-\left((m-2)^{2} / 4\right)$ and set $\delta=(m-2)^{2}+4 c$. Then the general solution of (2.3) (i) is

$$
q(r)=A r^{(2-m+\delta) / 2}+B r^{(2-m-\delta) / 2} .
$$

(b) Let $c=-\left((m-2)^{2} / 4\right)$. Then

$$
q(r)=A r^{1-m / 2}+B(\ln r) r^{1-m / 2} .
$$

(c) Let $c<-\left((m-2)^{2} / 4\right)$. Then $\delta=i k(k>0)$ and

$$
q(r)=A r^{k-m / 2} \sin \left(\frac{k}{2} \ln r\right)+B r^{1-m / 2} \cos \left(\frac{k}{2} \ln r\right) .
$$

Hence the functions

$$
\begin{cases}q=r^{\alpha} & \alpha \geqq 0 \\ g=r^{\gamma+m-3} & \gamma=2 \alpha \geqq 0\end{cases}
$$

satisfy (2.3). Indeed, since $\alpha \geqq 0$ (2.3) (ii) holds. An easy calculation yields $p(u)=(\gamma+m-2) u+\rho^{\gamma+m-2}$, which is certainly convex.

Notice that for $g=r^{\beta-1}$ the domain $D^{*}=\mathscr{R}_{g, A}(\mathscr{D})$ in the plane is given by

$$
D^{*}=\left\{\left(r, \theta_{1}\right) ; 0 \leqq r \leqq R^{*}\left(\theta_{1}\right),-\pi \leqq \theta_{1}<\pi\right\}
$$

where

$$
R^{*}(\theta)=\left\{\begin{array}{lll}
\left\{\sum_{j=1}^{n} a_{j} R_{j}^{\beta}\right\}^{1 / \beta} & \text { if } & \beta \neq 0 \\
\prod_{j=1}^{n} R_{j}^{a} j & \text { if } & \beta-0
\end{array}\right.
$$

and $R_{j}$ is defined in (1.8) with respect to $D_{j}$.

We mention also that for $g=r^{\beta-1}(\beta \geqq 0)$ in the plane, the inequality (2.11) may be replaced by:

$$
r_{0}^{*} \geqq \begin{cases}\left(\sum_{j=1}^{n} a_{j} r_{0, j}^{\beta}\right)^{1 / \beta} & \text { if } \beta>0 \\ \prod_{j=1}^{n} r_{0, j}^{a_{j}} & \text { if } \beta=0\end{cases}
$$

The inequality for $\beta=0$ was proved in [5]. For $\beta>0,(2.11)^{\prime}$ is obtained from (2.11) as follows. Set $\widetilde{D}_{j}=\left(1 / r_{0, j}\right) D_{j},(j=1, \cdots, n)$. Then the conformal radius of $\widetilde{D}_{j}$ at the origin is 1 . Let $\widetilde{D}^{*}=$ $\mathscr{R}_{g, A}{ }^{\prime}\left(\widetilde{D}_{1}, \cdots, \widetilde{D}_{n}\right)$, where $A^{\prime}=\left\{a_{1}^{\prime}, \cdots, a_{n}^{\prime}\right\}$ and $a_{k}^{\prime}=a_{k} r_{0, k}^{\beta} / \sum_{j=1}^{n} a_{j} r_{0, j}^{\beta}$. 
If $\widetilde{r}^{*}$ denotes conformal radius of $\widetilde{D}^{*}$ at the origin, we have (by (2.11)) $\widetilde{r}^{*} \geqq 1$. But $\widetilde{D}^{*}=\left[\left(\sum_{1}^{n} a_{j} r_{0, j}^{\beta}\right)^{-/ \beta}\right] D^{*}$. Hence $\widetilde{r}^{*}=r_{0}^{*} \quad\left(\sum_{1}^{n} a_{j} r_{0, j}^{\beta}\right)^{-1 / \beta} \geqq 1$.

Theorem 2.2 does not hold for $g=r^{-n-1}$ with $n=2,3, \cdots$. This is shown by the following counterexample. Let $D$ be the Koebe domain i.e., the entire $z$-plane cut along the positive real axis from $z=1 / 4$ to infinity. Set $D_{1}=D, D_{2}=e^{i 2 \pi} /{ }^{3} D$ and $D_{3}=e^{i \pi \pi} /{ }^{3} D$. Let $D^{*}=\mathscr{R}_{g, A}\left(D_{1}, D_{2}, D_{3}\right)$ with $g$ as above and $A=\{1 / 3,1 / 3,1 / 3\}$. Then $D^{*}$ is the entire $z$-plane, cut along the rays $\arg z=0,(2 \pi) / 3,(4 \pi) / 3$ from $|z|=\sqrt[n]{3 / 4}$ to infinity. But for $n=2,3, \cdots$ we have $\sqrt[n]{3 / 4}<$ $\sqrt[3]{1 / 4}$. Hence $r_{0}^{*}<1$ while $r_{0,1}=r_{0,2}=r_{0,3}=1$. This contradicts (2.11).

It is possible, of course, that (2.11) is valid for other families of functions $g$. In fact, examining the argument that proves Lemma 2.1 and Theorem 2.1 we observe that condition (2.3) is too restrictive. This condition (part (i)) guarantees that the integral

$$
\int_{\Omega} \int h^{2} q \Delta q d x d y
$$

is preserved under our transformation. But actually we need only that this integral does not decrease.

Furthermore, even if (2.11) does not hold for a given metric $g$ for every family of domains, it might hold for certain types of domains.

Finally we shall indicate an application of Theorem 1.1 concerning the harmonic radius. This notion was introduced by Hersch [2] and is defined in the following way: Let $D$ be a domain such that the Green function $g(P, Q)$ for the Laplace operator exists. The harmonic radius $R_{Q}$ is given by

$$
\frac{1}{R_{Q}}=\lim _{P \rightarrow Q}\left(4 \pi g(P, Q)-\frac{1}{|P Q|}\right)
$$

where $|P Q|$ is the distance between $P$ and $Q$. Following [6] we can characterize $R_{Q}$ with the help of the capacity $C_{\varepsilon}(Q)=\int_{\Omega \varepsilon}|\nabla \omega|^{2} d x$ where $\Omega_{\varepsilon}=D-\{P \in D|| P Q \mid<\varepsilon\} . \quad R_{Q}$ can be written as

$$
R_{Q}=\lim _{\varepsilon \rightarrow 0}\left\{4 \pi C_{\varepsilon}^{-1}(Q)-\frac{1}{\varepsilon}\right\}^{-1} .
$$

LEMMA 2.3. Let $\left\{D_{1}, D_{2}, \cdots, D_{n}\right\}=\mathscr{D}$ be a family of open sets in $R^{3}$ each containing the sphere $\{|x| \leqq \rho\}$, and let $R_{0, j}$ be the corresponding harmonic radius with respect to the origin. If $g=1$ and $D^{*}$ is defined by $D^{*}=R_{g, A}(\mathscr{D})$ [cf. Definition 1.3], then we have for the harmonic radius $R_{0}^{*}$ of $D^{*}$ at the origin

$$
\sum_{j=1}^{n} a_{j} R_{0, j} \leqq R_{0}^{*}
$$


Proof. From (2.13) we have

$$
C_{\varepsilon_{j}}(0)=C_{\varepsilon_{j}}=4 \pi\left(\frac{1}{R_{0, j}+1 / \varepsilon_{j}+O\left(\varepsilon_{j}\right)}\right),
$$

and by Theorem 2.1

$$
\begin{aligned}
\frac{1}{R_{0}^{*}+1 / \varepsilon+O(\varepsilon)} & =I\left(\Omega_{\varepsilon}^{*}\right) \leqq \sum_{j=1}^{n} a_{j} I\left(\Omega_{j, j}\right) \\
& =\sum_{j=1}^{n} \frac{a_{j}}{R_{j}+1 / \varepsilon_{j}+O\left(\varepsilon_{j}\right)}
\end{aligned}
$$

$\left[\Omega_{j, \varepsilon_{j}}=D_{j}-\left\{P \in D_{j}|| P O \mid<\varepsilon_{j}\right\}\right]$.

If we choose $\varepsilon_{j}=\left(\varepsilon R_{j} / \sum_{j=1}^{n} a_{j} R_{j}\right)=\beta R_{j}$. Then it follows from (2.14) that

$$
\frac{\varepsilon}{\varepsilon R_{0}^{*}+1+O\left(\varepsilon^{2}\right)} \leqq \sum_{j=1}^{n} a_{j} \frac{\beta R_{j}}{\beta R_{j}^{2}+1+O\left(\varepsilon^{2}\right)} .
$$

The function $\left(\beta x /\left(\beta x^{2}+1\right)\right)$ is concave in the interval $[0, m]$ where $m^{2} \leqq 3 / \beta$. Since $\varepsilon>0$ (and therefore $\beta$ ) is arbitrary, it is always possible to find a number $\beta_{0}$ such that $\left(\beta_{0} x /\left(\beta_{0} x^{2}+1\right)\right)$ is concave in $\left[0, \max _{j} R_{j}\right]$. Therefore

$$
\begin{aligned}
\sum_{j=1}^{n} a_{j} \frac{\beta_{0} R_{j}}{\beta_{0} R_{j}^{2}+1+O\left(\varepsilon^{2}\right)} & \leqq \sum_{j=1}^{n} \frac{\beta_{0}\left(\sum_{j} a_{j} R_{j}\right)}{\beta 0\left(\sum_{j} a_{j} R_{j}\right)^{2}+1+O\left(\varepsilon^{2}\right)} \\
& =\sum_{j=1}^{n} \frac{\varepsilon_{0}}{\beta_{0}\left(\sum_{j} a_{j} R_{j}\right)+1+O(1)}
\end{aligned}
$$

and by (2.16) we have

$$
R_{0}^{*} \geqq \sum_{j=1}^{n} a_{j} R_{j}
$$

\section{REFERENCES}

1. W. K. Hayman, Multivalent Functions, Cambridge University Press.

2. J. Hersch, Transplantation harmonique, transplantation par modules et théorèmes isopérimétriques, Comment. Math. Helv., 44 (1969), 354-366.

3. R. Kühnau, Uber schraubungssymmetrische Potentialfelder, Math. Nachrichten, 45 (1970), 345-351.

4. M. Marcus, Transformations of domains in the plane and applications in the theory of functions, Pacific J. Math., 14 (1964), 613-626.

5. - Radial averaging of domains, estimates for Dirichlet integrals and applications, Carnegie-Mellon University, Technical Report 71-36, (to appear in J. D'Anal. Math.).

6. G. Pólya and G. Szegö, Isoperimetric Inequalities in Mathematical Physics, Princeton University Press, 1951.

7. G. Szegö, On a certain kind of symmetrization and its applications, Ann. Mat. Pura ed Applicata, Ser. 4. 40 (1955), 113-119. 
8. G. Szegö, Uber eine Verallgemeinerung des Dirichletschen Integrals, Math. Z., 52 (1950), 676-685.

Received October 7, 1971 and in revised form November 3, 1972.

\section{CARNEGIE-MELLON UNIVERSity}

Present Adresses:

C. BANDLE, Stanford University Stanford, Calif. 94305

M. Marcus, Technion, Haifa, Israel 


\section{PACIFIC JOURNAL OF MATHEMATICS}

\section{EDITORS}

D. Gilbarg and J. Milgram

Stanford University

Stanford, California 94305

\author{
R. A. Beaumont \\ University of Washington \\ Seattle, Washington 98105
}

J. DuGUNDJI

Department of Mathematics

University of Southern California

Los Angeles, California 90007

Richard ARENS

University of California

Los Angeles, California 90024

\section{ASSOCIATE EDITORS}
E. F. BECKENBACH
B. H. NeumanN
F. WOLF
K. YosHIDA

\section{SUPPORTING INSTITUTIONS}

UNIVERSITY OF BRITISH COLUMBIA

CALIFORNIA INSTITUTE OF TECHNOLOGY

UNIVERSITY OF CALIFORNIA

MONTANA STATE UNIVERSITY

UNIVERSITY OF NEVADA

NEW MEXICO STATE UNIVERSITY

OREGON STATE UNIVERSITY

UNIVERSITY OF OREGON

OSAKA UNIVERSITY

\author{
UNIVERSITY OF SOUTHERN CALIFORNIA \\ STANFORD UNIVERSITY \\ UNIVERSITY OF TOKYO \\ UNIVERSITY OF UTAH \\ WASHINGTON STATE UNIVERSITY \\ UNIVERSITY OF WASHINGTON
* * * *
AMERICAN MATHEMATICAL SOCIETY \\ NAVAL WEAPONS CENTER
}

The Supporting Institutions listed above contribute to the cost of publication of this Journal, but they are not owners or publishers and have no responsibility for its content or policies.

Mathematical papers intended for publication in the Pacific Journal of Mathematics should be in typed form or offset-reproduced, (not dittoed), double spaced with large margins. Underline Greek letters in red, German in green, and script in blue. The first paragraph or two must be capable of being used separately as a synopsis of the entire paper. The editorial "we" must not be used in the synopsis, and items of the bibliography should not be cited there unless absolutely necessary, in which case they must be identified by author and Journal, rather than by item number. Manuscripts, in duplicate if possible, may be sent to any one of the four editors. Please classify according to the scheme of Math. Rev. Index to Vol. 39. All other communications to the editors should be addressed to the managing editor, Richard Arens, University of California, Los Angeles, California, 90024.

50 reprints are provided free for each article; additional copies may be obtained at cost in multiples of 50 .

The Pacific Journal of Mathematics is issued monthly as of January 1966. Regular subscription rate: $\$ 48.00$ a year (6 Vols., 12 issues). Special rate: $\$ 24.00$ a year to individual members of supporting institutions.

Subscriptions, orders for back numbers, and changes of address should be sent to Pacific Journal of Mathematics, 103 Highland Boulevard, Berkeley, California, 94708.

PUBLISHED BY PACIFIC JOURNAL OF MATHEMATICS, A NON-PROFIT CORPORATION

Printed at Kokusai Bunken Insatsusha (International Academic Printing Co., Ltd.), 270, 3-chome Totsuka-cho, Shinjuku-ku, Tokyo 160, Japan. 


\section{Pacific Journal of Mathematics}

\section{Vol. 46, No. 2 December, 1973}

Christopher Allday, Rational Whitehead products and a spectral sequence of

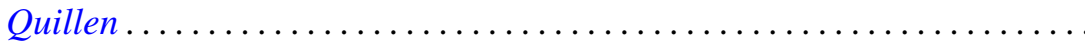

James Edward Arnold, Jr., Attaching Hurewicz fibrations with fiber

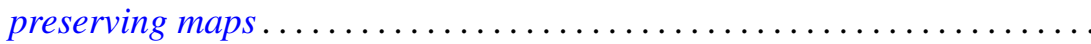

Catherine Bandle and Moshe Marcus, Radial averaging transformations with various metrics.................................

David Wilmot Barnette, A proof of the lower bound conjecture for convex

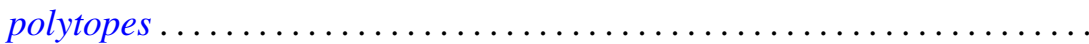

Louis Harvey Blake, Simple extensions of measures and the preservation of

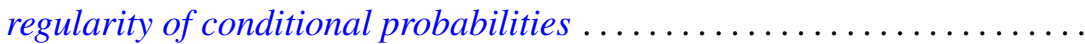

James W. Cannon, New proofs of Bing's approximation theorems for

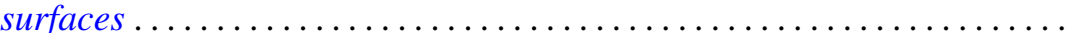

C. D. Feustel and Robert John Gregorac, On realizing HNN groups in

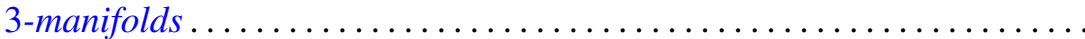

Theodore William Gamelin, Iversen's theorem and fiber algebras . . . . . . . . 389

Daniel H. Gottlieb, The total space of universal fibrations . . . . . . . . . . . .

Yoshimitsu Hasegawa, Integrability theorems for power series expansions of

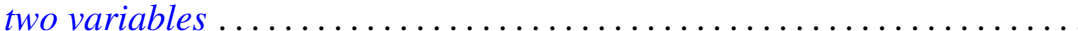

Dean Robert Hickerson, Length of period simple continued fraction expansion of $\sqrt{ } d$

Herbert Meyer Kamowitz, The spectra of endomorphisms of the disc algebra.

Dong S. Kim, Boundedly holomorphic convex domains

Daniel Ralph Lewis, Integral operators on $\mathscr{L}_{p}$-spaces ...

John Eldon Mack, Fields of topological spaces . . . . . . . . .

V. B. Moscatelli, On a problem of completion in bornology

Ellen Elizabeth Reed, Proximity convergence structures. .

Ronald C. Rosier, Dual spaces of certain vector sequence spaces .

Robert A. Rubin, Absolutely torsion-free rings

Leo Sario and Cecilia Wang, Radial quasiharmonic functions . .

James Henry Schmerl, Peano models with many generic classes .

H. J. Schmidt, The $\mathscr{F}$-depth of an $\mathscr{F}$-projector ............

Edward Silverman, Strong quasi-convexity. . . . . . . . . . . . . . . . . 549

Barry Simon, Uniform crossnorms ....................... 555

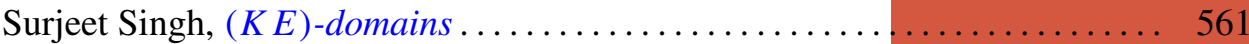

Ted Joe Suffridge, Starlike and convex maps in Banach spaces . . . . . . . . 575

Milton Don Ulmer, $C$-embedded $\Sigma$-spaces . . . . . . . . . . . . . . . . 591

Wolmer Vasconcelos, Conductor, projectivity and injectivity . . . . . . . . . 603 